Article

\title{
Classification and Characterization of Tire-Road Wear Particles in Road Dust by Density
}

\author{
Uiyeong Jung and Sung-Seen Choi *
}

check for updates

Citation: Jung, U.; Choi, S.-S Classification and Characterization of Tire-Road Wear Particles in Road Dust by Density. Polymers 2022, 14, 1005. https://doi.org/10.3390/ polym14051005

Academic Editors: Sang-Eun Shim, Jeong Seok Oh and Seung Hyun Lee

Received: 29 January 2022

Accepted: 25 February 2022

Published: 2 March 2022

Publisher's Note: MDPI stays neutral with regard to jurisdictional claims in published maps and institutional affiliations.

Copyright: (C) 2022 by the authors. Licensee MDPI, Basel, Switzerland. This article is an open access article distributed under the terms and conditions of the Creative Commons Attribution (CC BY) license (https:// creativecommons.org/licenses/by/ $4.0 /)$.
Department of Chemistry, Sejong University, 209 Neungdong-ro, Gwangjin-gu, Seoul 05006, Korea; dmldud3731@sju.ac.kr

* Correspondence: sschoi@sejong.ac.kr

\begin{abstract}
Tire treads are abraded by friction with the road surface, producing tire tread wear particles (TWPs). TWPs combined with other particles on the road such as road wear particles (RWPs) and mineral particles (MPs), forming tire-road wear particles (TRWPs). Dust on an asphalt pavement road is composed of various components such as TRWPs, asphalt pavement wear particles (APWPs), MPs, plant-related particles (PRPs), and so on. TRWPs have been considered as one of major contaminants produced by driving and their properties are important for study on real abrasion behaviors of tire treads during driving as well as environmental contamination. Densities of the TRWPs are totally dependent on the amount of the other components deposited in the TWPs. In this study, a classification method of TRWPs in the road dust was developed using density separation and the classified TRWPs were characterized using image analysis and pyrolytic technique. Chloroform was used to remove APWPs from mixture of TRWPs and APWPs. TRWPs were found in the density range of $1.20-1.70 \mathrm{~g} / \mathrm{cm}^{3}$. By decreasing the particle size of the road dust, the TRWP content in the road dust increased and its density slightly tended to increase. Aspect ratios of the TRWPs varied and there were many TRWPs with low aspect ratio below 2.0. The aspect ratio range was 1.2-5.2. Rubber compositions of the TRWPs were found to be mainly NR/SBR biblend or NR/BR/SBR triblend.
\end{abstract}

Keywords: tire-road wear particle; road dust; density separation; characterization; asphalt pavement wear particle

\section{Introduction}

Tire treads are abraded by friction between the road surface and the tire tread, resulting in the formation of tire-road wear particles (TRWPs) [1,2]. TRWPs are usually black particles with a stick or round shape, and, to some extent, they are encrusted by mineral and other tiny particles [3,4]. Since rubber has a slightly sticky surface [5], such particles can easily adhere to the rubber particle (tire tread wear particle, TWP) surface. When contaminants attach to the TWP surface, they can release to environment. Especially, the particles of small size float in the air to cause air pollution as fine dust or they enter the aquatic environment to cause water pollution [6]. Therefore, TRWPs have been considered a kind of microplastics and a pollutant $[7,8]$.

In general, densities of the TWPs are about $1.2 \mathrm{~g} / \mathrm{cm}^{3}$, while the TRWP densities should be higher than the TWPs due to the encrusted mineral particles $[9,10]$. Granite and marble are some of representative rock components and their densities are about $2.7 \mathrm{~g} / \mathrm{cm}^{3}[11,12]$. TRWPs with high density have many mineral particles or environmental contaminants attached to the surface. Since TRWPs have various densities, it is necessary to subdivide TRWPs by density and analyze their properties. Tire tread wear is also directly related to the lifespan of a tire, so careful and detailed researches on the abrasion behaviors are required. Real road dust is composed of various components including TRWPs, asphalt pavement wear particles (APWPs), mineral particles (MPs), glass particles (GPs), glass beads (GBs), plant-related particles (PRPs), road paint wear particles (RPWPs), plastic 
particles (PPs), and others [4]. Hence, TRWPs should be separated from the road dust at first to examine properties of TRWPs.

A density separation method has been employed for separation of TRWPs from the environmental samples, and it uses the density differences of the components. It was reported that a very high-density medium of sodium polytungstate solution (density: $2.2 \mathrm{~g} / \mathrm{cm}^{3}$ ) was used for the density separation [1]. Using this method, the TRWPs were found in the floated fraction but lots of other particles also existed in the same fraction. Klockner et al. used an instrument consisting of a $50 \mathrm{~mL}$ volumetric flask equipped with PVC tube and sodium polytungstate solution of $1.9 \mathrm{~g} / \mathrm{cm}^{3}$, and they could fractionate the sample 1 day after the solution treatment [2]. This method may be semi-automated using the instrument but it is somewhat complex and takes a long time. To effectively separate TRWPs from the environmental samples and determine their detailed densities, it is required to develop a new method. In this study, a separation method of TRWPs from the environmental sample of road dust was developed using salt solutions with different densities and chloroform. Chloroform was used to eliminate APWPs from the sample mixed with TRWPs. The TRWPs were classified by $0.10 \mathrm{~g} / \mathrm{cm}^{3}$ density unit and their structural characteristics and rubber compositions were analyzed. We believe that this analytical method could be also applied to various environmental samples containing TRWPs such as sediments of river and sea.

\section{Materials and Methods}

\subsection{Materials}

Sodium bromide (purity: 99.0\%) and sodium iodide (purity: 99.0\%) were purchased from Ducksan Co. (Ansan, Korea). Chloroform (purity: 99.5\%) was purchased from Samchun Chemical Co. (Seoul, Korea). Deionized water was used to prepare the salt solutions.

\subsection{Matrix Solution Preparation for Density Separation}

For density separation, salt solutions and chloroform were used. Sodium bromide and sodium iodide were used for the preparation of the salt solutions in deionized water. Density of the saturated sodium bromide solution was $1.52 \mathrm{~g} / \mathrm{cm}^{3}$ at $22{ }^{\circ} \mathrm{C}$. Sodium bromide solutions with densities of 1.20, 1.30, 1.40 and $1.50 \mathrm{~g} / \mathrm{cm}^{3}$ were prepared. For the preparation of higher density solutions, sodium iodide was used. Density of the saturated solution of sodium iodide was $1.83 \mathrm{~g} / \mathrm{cm}^{3}$ at $24{ }^{\circ} \mathrm{C}$. Sodium iodide solutions with densities of 1.60 and $1.70 \mathrm{~g} / \mathrm{cm}^{3}$ were prepared. Density of chloroform is $1.49 \mathrm{~g} / \mathrm{cm}^{3}$.

\subsection{Preparation of Road Dust Sample}

Road dust was collected in January 2021 near a bus stop by sweeping with a broom. The road dust was separated by size $(212-500,106-212$, and 63-106 $\mu \mathrm{m})$ using a sieve shaker of Octagon 200 (Endecotts Co., London, UK). Morphology of the particles was analyzed using an image analyzer (EGVM 35B, EG Tech., Anyang, Korea).

In order to examine components of the road dust, all of the particles in $10 \mathrm{mg}$ road dust of 212-500 $\mu \mathrm{m}$ (sample code: RD1) were classified into the kinds with the naked eye under a microscope. Rough density of each particle was also measured using the salt solutions of 1.2 and $1.6 \mathrm{~g} / \mathrm{cm}^{3}$. The road dust samples of $212-500 \mu \mathrm{m}(20 \mathrm{mg}$, sample code:

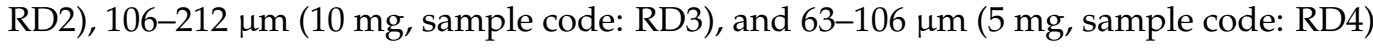
were used.

\subsection{Density Separation Method}

Approximately $50 \mathrm{~mL}$ of salt solution or chloroform was put into a glass container (diameter $70 \mathrm{~mm}$, height $40 \mathrm{~mm}$ ), and the road dust sample was put into the container. In the case of using chloroform, the sonication (ultrasonic cleaner, Jeiotech Co., Daejeon, Korea) process was performed for an additional $10 \mathrm{~min}$. In order to sufficiently wet the particles, the solution was dropped several times with a micro pipette and left for $30 \mathrm{~min}$. 
After this process was repeated twice, the particles sunk and floated were separated using a micro pipette. The separated particles were transferred to a petri dish, washed 3 times with deionized water and finally washed once more with ethanol, and then dried at room temperature.

\subsection{Characterization of TRWPS}

Morphology analysis of the classified TRWPs was performed using a digital microscope (Leica DM4 M, Leica Microsystems, Wetzlar, Germany). Lengths of the major and minor axes were measured using the 2D analysis module, and the aspect ratio was obtained. Other tiny particles deposited on the TRWP surface were also observed.

Rubber composition of the TRWPs was analyzed using pyrolysis-gas chromatography (Py-GC). YL6500GC (Younglin Co., Seoul, Korea) equipped with CDS 2000 Pyroprobe (CDS Analytical Inc., Oxford, PA, USA) was used. The interface temperature of the pyrolyzer was $250{ }^{\circ} \mathrm{C}$. The sample was put in a quartz tube and both sides were covered with glass wool and then pyrolyzed at $520{ }^{\circ} \mathrm{C}$ for $10 \mathrm{~s}$. Temperature of the GC injector was $250{ }^{\circ} \mathrm{C}$. An HP-5 column ( $30 \mathrm{~m} \times 0.25 \mathrm{~mm}, 0.25 \mu \mathrm{m}$ thickness) was used. The split ratio was 1:15 and $\mathrm{N}_{2}$ was used as the carrier gas. The GC oven temperature program was as follows: $30{ }^{\circ} \mathrm{C}$ (held for $3 \mathrm{~min}$ ) to $180{ }^{\circ} \mathrm{C}$ (held for $1 \mathrm{~min}$ ) at $10{ }^{\circ} \mathrm{C} / \mathrm{min}$, then to $250{ }^{\circ} \mathrm{C}$ (held for $3 \mathrm{~min}$ ) at $10{ }^{\circ} \mathrm{C} / \mathrm{min}$.

\section{Results and Discussion}

\subsection{Composition and Density Properties of the Road Dust}

In order to examine the kinds of components in the road dust, each particle in $10 \mathrm{mg}$ road dust of 212-500 $\mu \mathrm{m}$ was classified and their densities were measured. Among the black particles, elastic particles with elongated or round shapes were classified into TRWPs. On the other hand, APWPs were selected based on the feature of being inelastic and breaking when pressed.

Besides TRWPs and APWPs, there were various kinds of particles in the road dust samples such as MPs, GPs, GBs, RPWPs, PRPs, and PPs as listed in Table 1. MPs were most abundant in the road dust, and they had a rigid and angular shape. PPs were thin and slightly hard. RPWPs were particles produced by wearing road marking paint, and they were white or yellow color and crumbled when pressed. PRPs were generated from plants around the bus stop, and they could be crushed into smaller pieces. GPs were transparent and angular shape, whereas GBs were spherical.

Table 1. Particle components of the RD1 sample $(212-500 \mu \mathrm{m})$.

\begin{tabular}{cc}
\hline Particle & Ratio (wt $\mathbf{\%})$ \\
\hline Tire-road wear particles (TRWPs) & 0.5 \\
Asphalt pavement wear particles (APWPs) & 7.0 \\
Mineral particles (MPs) & 77.2 \\
Glass particles (GPs) & 11.7 \\
Glass beads (GBs) & 1.5 \\
Road paint wear particles (RPWPs) & 1.1 \\
Plant-related particles (PRPs) & 0.9 \\
Plastic particles (PPs) & 0.1 \\
\hline
\end{tabular}

Rough densities of the classified particles of the RD1 sample are summarized in Figure 1. Densities of TRWPs, RPWPs, and PRPs were widely spread over $1.2 \mathrm{~g} / \mathrm{cm}^{3}$, while those of APWPs, MPs, GPs, and GBs were over $1.6 \mathrm{~g} / \mathrm{cm}^{3}$. Density of PPs was lower than $1.6 \mathrm{~g} / \mathrm{cm}^{3}$. Based on these results, the reference density was set to $1.6 \mathrm{~g} / \mathrm{cm}^{3}$. When the road dust sample is put in the matrix solution with density of $1.6 \mathrm{~g} / \mathrm{cm}^{3}$, PPs will be floated while MPs, APWPs, GPs, and GBs sink. For TRWPs, RPWPs, and RPPs, some of them can float and the others may sink. 


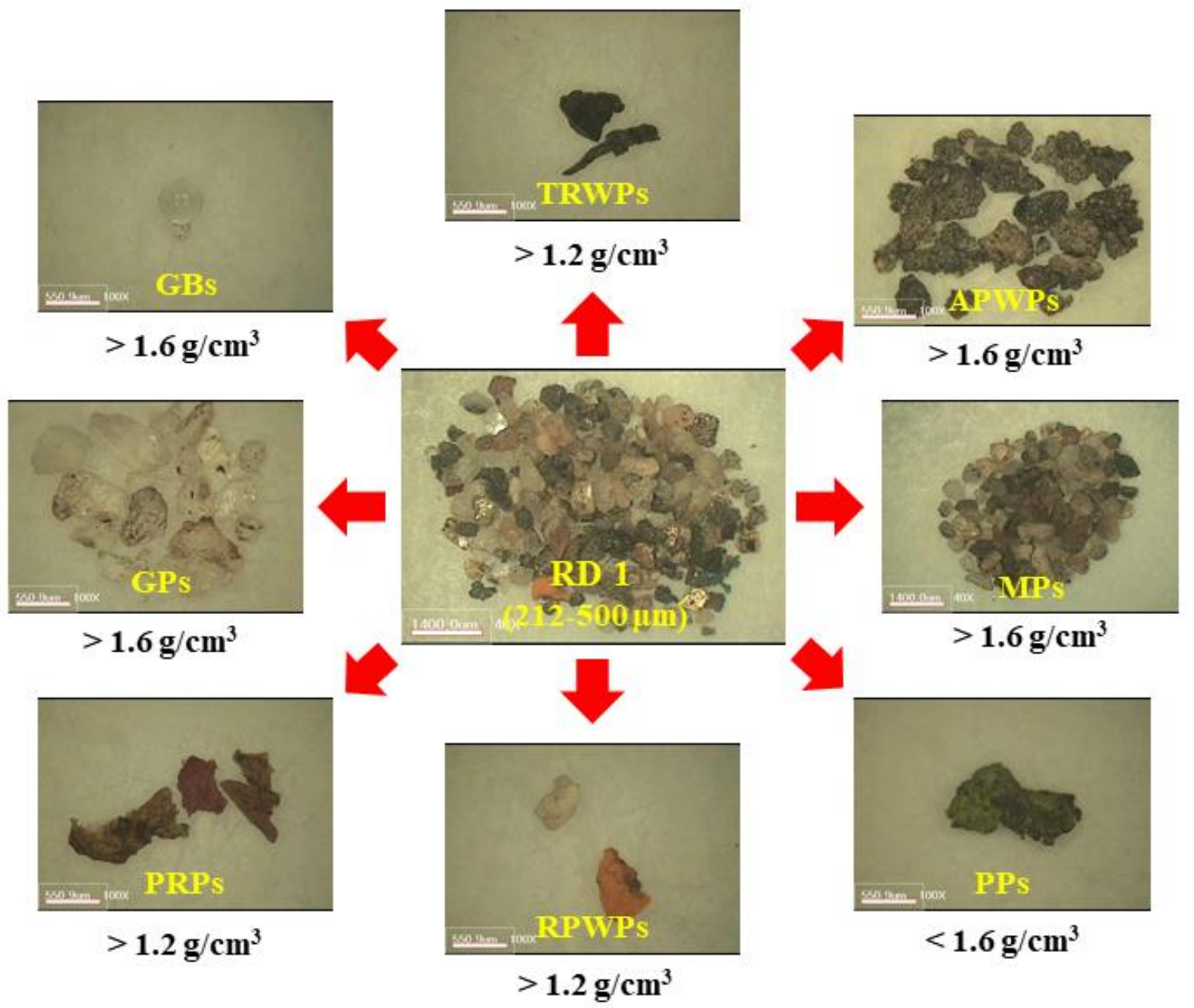

Figure 1. Density ranges of various particles in the RD1 sample. The scale bar of the center image is $1400 \mu \mathrm{m}$, and those of the others are $551 \mu \mathrm{m}$.

In order to examine the density distribution of the TRWPs by $0.10 \mathrm{~g} / \mathrm{cm}^{3}$ unit, $10 \mathrm{TR}$ WPs were randomly selected from the road dust of 106-212 $\mu \mathrm{m}$ because there were rare TRWPs in the road dust of $212-500 \mu \mathrm{m}$. The TRWPs had densities of $1.20-1.60 \mathrm{~g} / \mathrm{cm}^{3}$, and those with low density of $1.20-1.30 \mathrm{~g} / \mathrm{cm}^{3}$ were much greater than the others, as shown in Figure 2. Based on the density ranges of TRWPs, the density separation was carried out using the salt solutions with densities of 1.20,1.30,1.40,1.50, 1.60, and $1.70 \mathrm{~g} / \mathrm{cm}^{3}$.

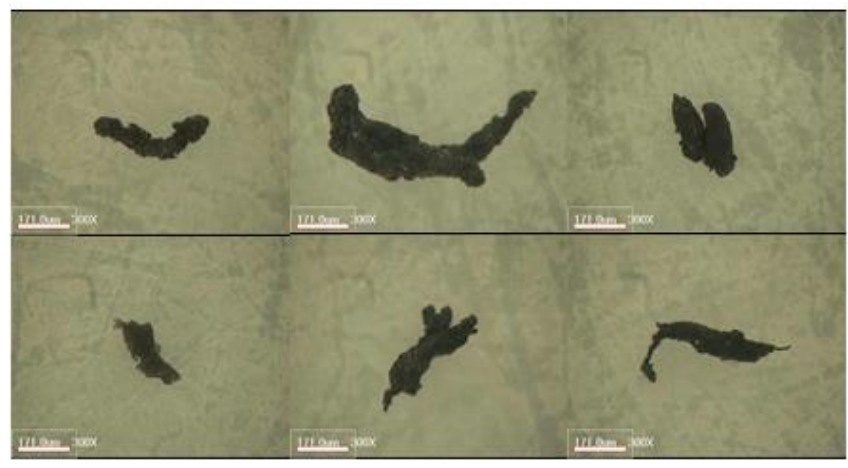

(a)

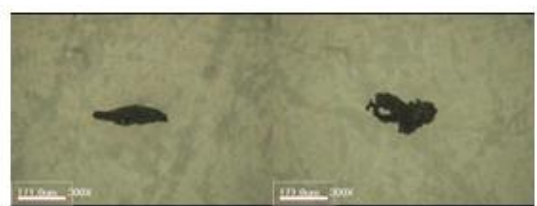

(b)

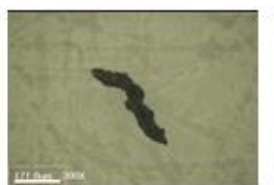

(c)

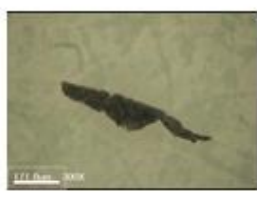

(d)

Figure 2. Magnified images of TRWPs selected from the road dust of 106-212 $\mu \mathrm{m}$. (a) $1.20-1.30 \mathrm{~g} / \mathrm{cm}^{3}$, (b) $1.30-1.40 \mathrm{~g} / \mathrm{cm}^{3}$, (c) $1.40-1.50 \mathrm{~g} / \mathrm{cm}^{3}$, and (d) $1.50-1.60 \mathrm{~g} / \mathrm{cm}^{3}$. The scale bars are $171 \mu \mathrm{m}$. 


\subsection{Differentiation of TRWPs and APWPS}

Shapes and colors of TRWP and APWP in road dust are very similar, and some TRWPs with high density are the same density region of APWPs. For this reason, it is hard to differentiate TRWPs from APWPs in the same density range. Fortunately, bitumen used as a binder of asphalt pavement is easily dissolved with some solvents such as chloroform, but crosslinked rubber does not dissolved with any solvents. In this study, TRWPs were differentiated from APWPs in the same density range using chloroform treatment. When an APWP is put into chloroform, bitumen part of the APWP is dissolved, as shown in Figure 3.

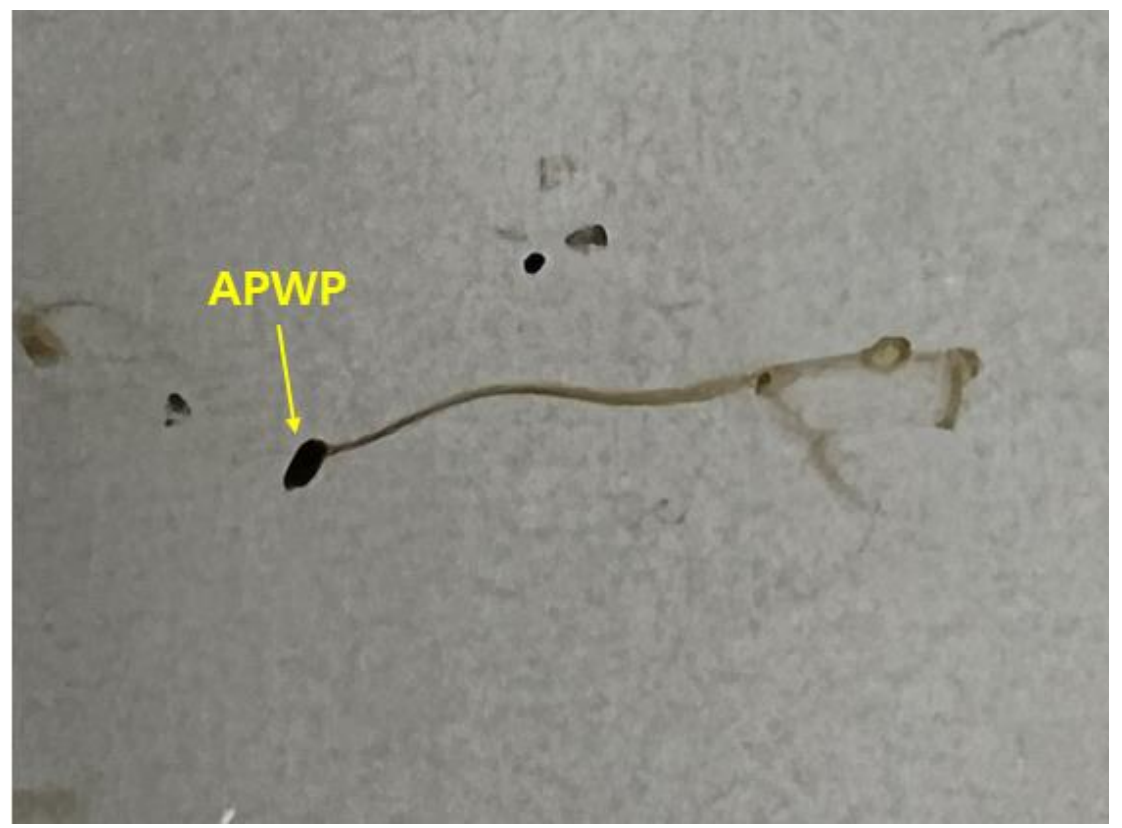

Figure 3. Dissolution of bitumen in APWP by chloroform.

A TRWP and an APWP with similar size and shape were selected, and chloroform was dropped on them (Figure 4). After the chloroform treatment, the TRWP still maintained its initial shape but the APWP split into pieces and the degree was more severe by increasing the chloroform drop. When the whole bitumen part of an APWP was totally dissolved in chloroform, the APWP shape disappeared and aggregate (broken stone) in the APWP was appeared. Hence, we can conclude that APWPs mixed with TRWPs are removed by chloroform treatment. By the chloroform treatment, binder components such as bitumen in APWPs dissolve and the stone components with high density settle down. 


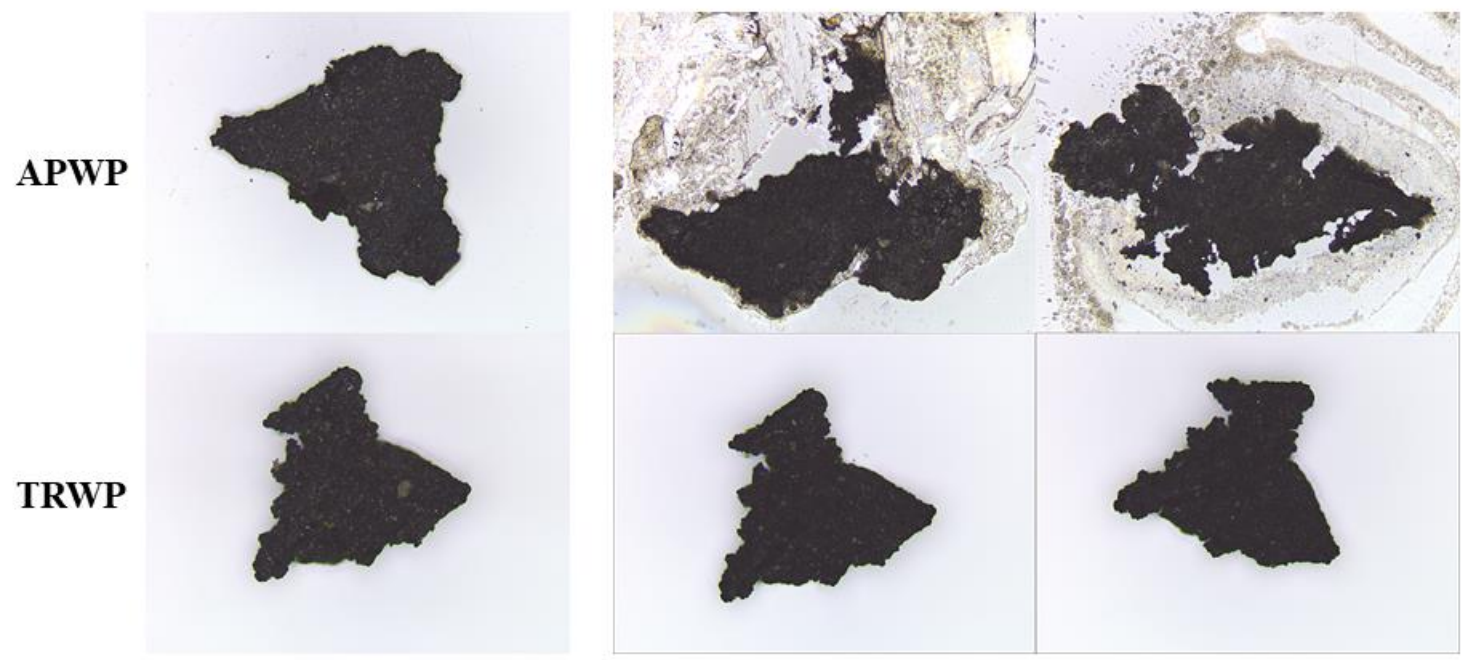

Figure 4. Difference in the shape changes of TRWP and APWP selected from the road dust of 212-500 $\mu \mathrm{m}$ after the chloroform treatment.

\subsection{Density Separation of the Road Dust Samples}

An experimental procedure for TRWP separation from road dust and classification of the TRWPs by density is described in Figure 5. First, the road dust sample was put into the salt solution of $1.60 \mathrm{~g} / \mathrm{cm}^{3}$ and divided into high- and low-density particles corresponding to the sunk and floating parts, respectively. Second, the high-density moiety was put into the salt solution of $1.70 \mathrm{~g} / \mathrm{cm}^{3}$, the floated 1 was treated with chloroform to remove APWPs, and TRWPs were selected from the mixture. The TRWPs selected from the second step have density of $1.60-1.70 \mathrm{~g} / \mathrm{cm}^{3}$. Third, the moiety floated in the salt solution of $1.60 \mathrm{~g} / \mathrm{cm}^{3}$ was put into the salt solution of $1.20 \mathrm{~g} / \mathrm{cm}^{3}$ to remove light particles such as some of PRPs and PPs. Fourth, the moiety sunk in the salt solution of $1.20 \mathrm{~g} / \mathrm{cm}^{3}$ was put into the salt solution of $1.40 \mathrm{~g} / \mathrm{cm}^{3}$ and the sunk and floated parts were put into the salt solutions of 1.50 and $1.30 \mathrm{~g} / \mathrm{cm}^{3}$, respectively. Fifth, the moieties sunk and floated in the salt solution of $1.50 \mathrm{~g} / \mathrm{cm}^{3}$ were divided into 2 groups of $1.50-1.60$ and $1.40-1.50 \mathrm{~g} / \mathrm{cm}^{3}$, respectively. The TRWPs selected from the fifth step have densities of $1.50-1.60$ and $1.40-1.50 \mathrm{~g} / \mathrm{cm}^{3}$, respectively. Finally, the moieties sunk and floated in the salt solution of $1.30 \mathrm{~g} / \mathrm{cm}^{3}$ were divided into 2 groups of $1.30-1.40$ and $1.20-1.30 \mathrm{~g} / \mathrm{cm}^{3}$, respectively. The TRWPs selected from the final step have densities of $1.30-1.40$ and $1.20-1.30 \mathrm{~g} / \mathrm{cm}^{3}$, respectively.

The numbers of TRWPs after the density separation were counted and the results have been summarized in Figure 6. There was no TRWP below $1.20 \mathrm{~g} / \mathrm{cm}^{3}$ or above $1.70 \mathrm{~g} / \mathrm{cm}^{3}$. For the RD2 sample of 212-500 $\mu \mathrm{m}$, TRWPs were rarely found and another road dust sample of 212-500 $\mu \mathrm{m}$ did not have any TRWP. There were only TRWPs with relatively low densities below $1.50 \mathrm{~g} / \mathrm{cm}^{3}$ in the RD2 sample. For the RD3 sample of 106-212 $\mu \mathrm{m}$, TRWPs with all density ranges of $1.20-1.70 \mathrm{~g} / \mathrm{cm}^{3}$ were found. There were relatively even TRWPs with density ranges of $1.30-1.70 \mathrm{~g} / \mathrm{cm}^{3}$ in the RD4 sample of 63-106 $\mu \mathrm{m}$. By decreasing the road dust size, the TRWP size tended to smaller. 


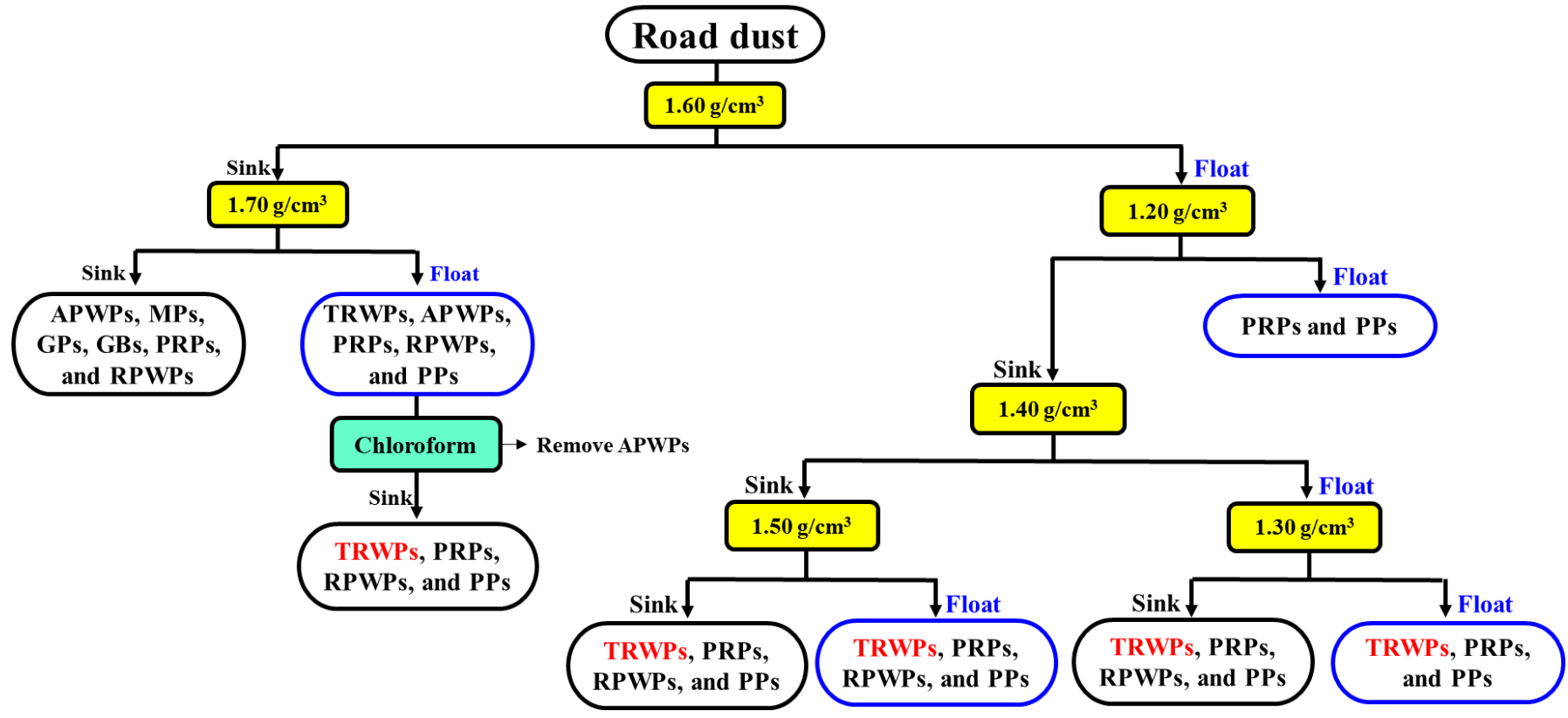

Figure 5. Separation procedure of TRWPs from road dust by the density difference.

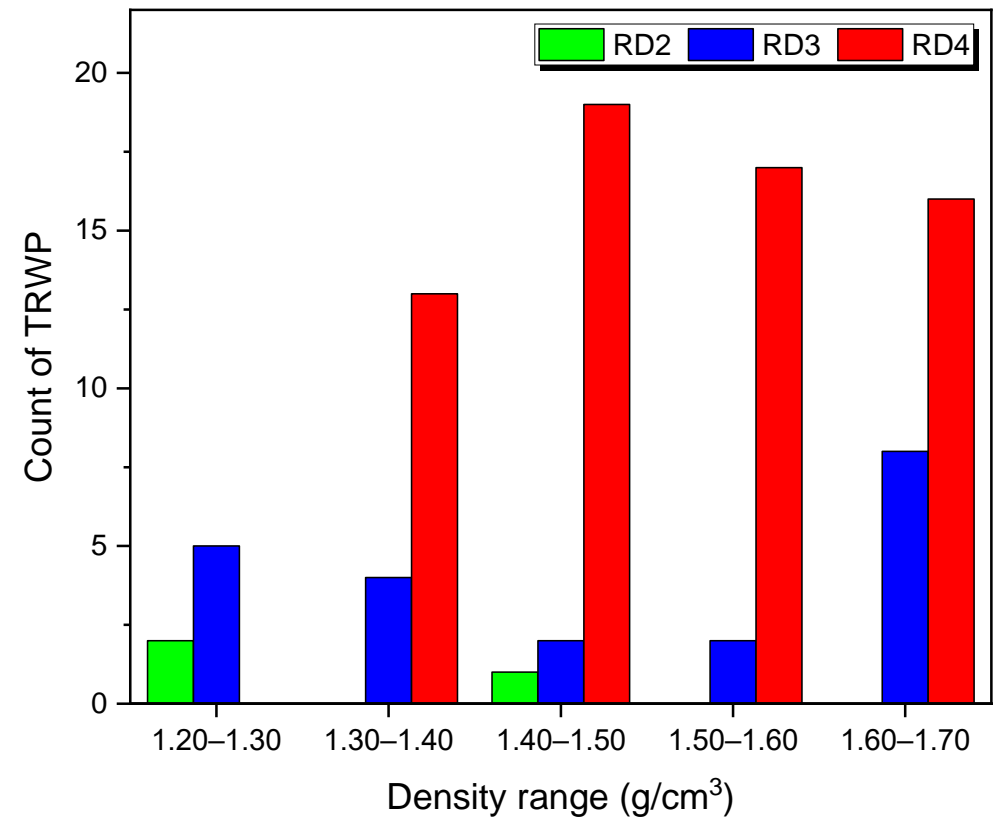

Figure 6. Variations in the numbers of TRWPs in the road dust samples with the TRWP density.

The contents of the TRWPs in the road dust samples are summarized in Figure 7. The TRWP content clearly increased as the road dust sample size decreased. This means that size distribution of TWPs produced by abrasion of tire treads shifted to smaller one or initial TRWPs were broken into smaller pieces by friction between the road and the tire. For the RD2 sample of 212-500 $\mu \mathrm{m}$, content of the TRWP with $1.20-1.30 \mathrm{~g} / \mathrm{cm}^{3}$ was much greater than that with $1.40-1.50 \mathrm{~g} / \mathrm{cm}^{3}$. For the RD3 sample of 106-212 $\mu \mathrm{m}$, content of the TRWP with 1.40-1.50 $\mathrm{g} / \mathrm{cm}^{3}$ was larger than those of the other sizes. Compared to the TRWPs with the same density range, the TRWP content tended to increase with decrease in the particle size. 


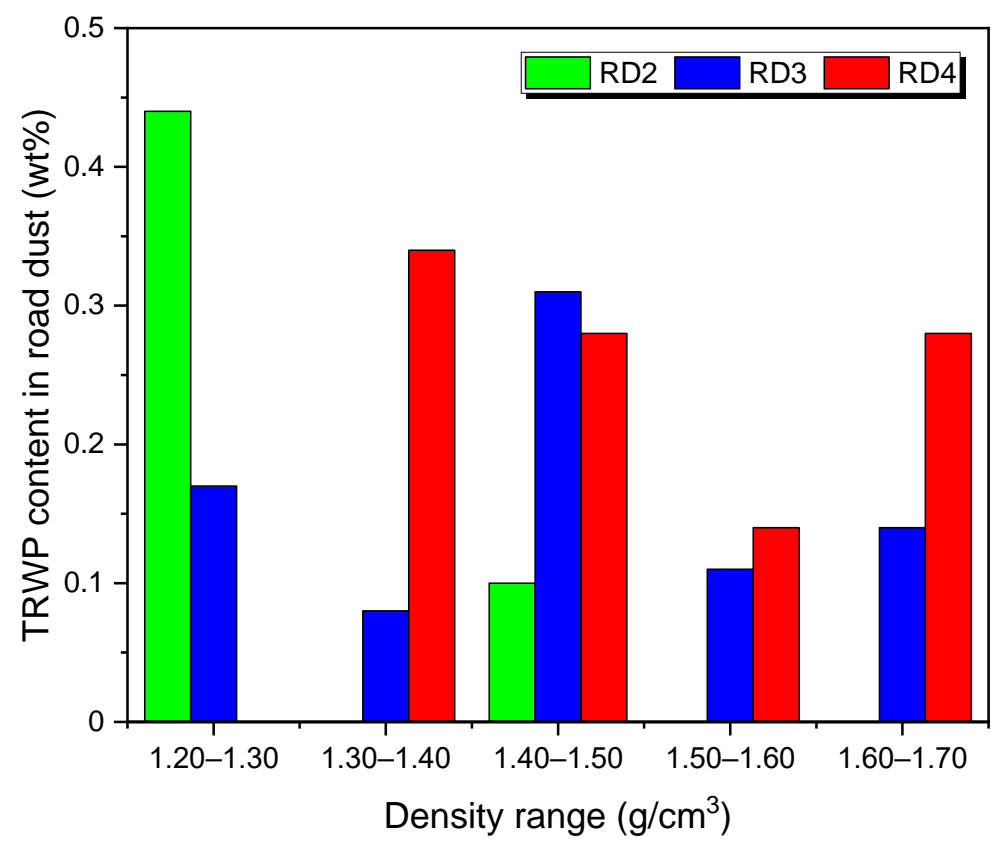

Figure 7. Variations in the TRWP contents in the road dust samples with the TRWP density.

\subsection{Characterization of TRWPS Classified by the Density Separation}

Shapes of the classified TRWPs were analyzed and the aspect ratios were measured. Magnified images of the representative TRWPs are shown in Figure 8. The TRWP surface was magnified to examine the tiny particles attached to the surface as shown in Figure 9. Particle sizes on the TRWP surface varied and the number of particles on the TRWP surface tended to increase as the TRWP density increased.

RD 2

RD 3
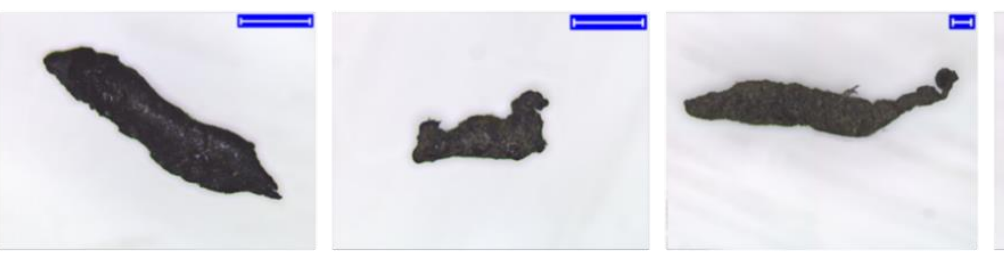

RD 4

No TRWPs
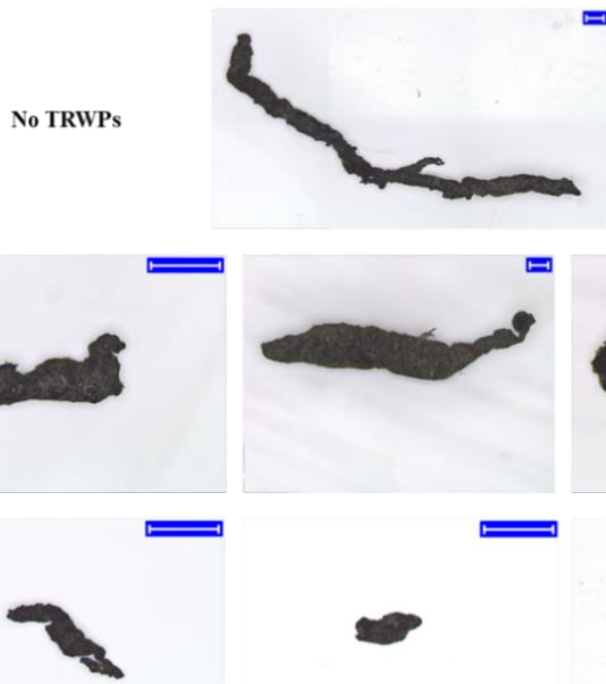

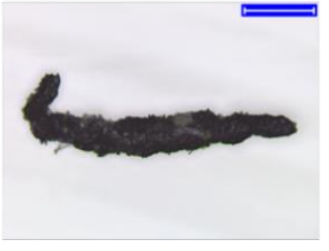

No TRWPs
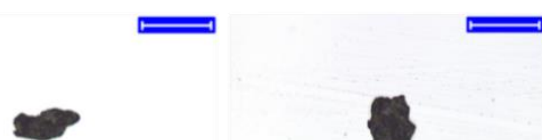

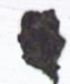

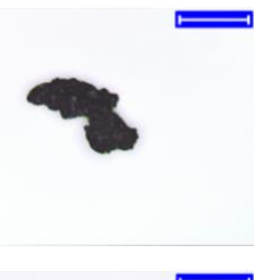

No TRWPs

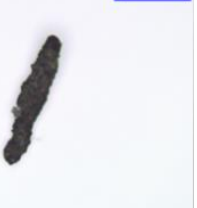

Figure 8. Magnified images of the TRWPs classified from the road samples. The blue scale bar is $100 \mu \mathrm{m}$ 
$1.20-1.30 \mathrm{~g} / \mathrm{cm}^{3}$

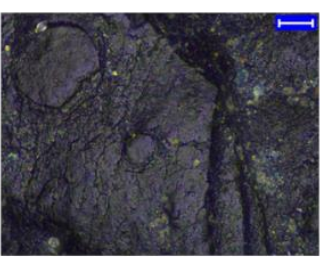

RD 3

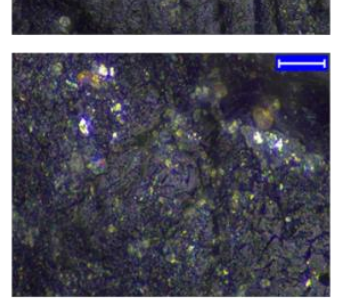

RD 4

RD 2
$1.30-1.40 \mathrm{~g} / \mathrm{cm}^{3}$

No TRWPs
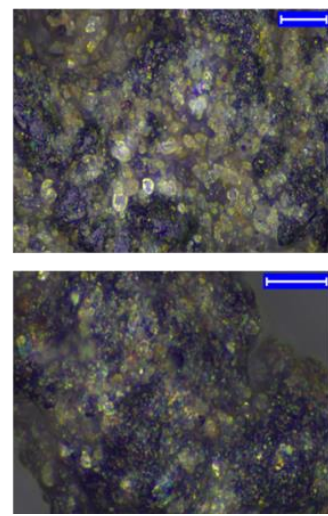

$1.40-1.50 \mathrm{~g} / \mathrm{cm}^{3}$
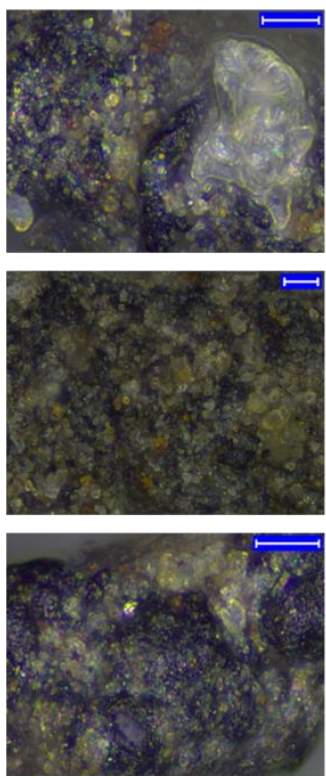

$1.50-1.60 \mathrm{~g} / \mathrm{cm}^{3}$

$1.60-1.70 \mathrm{~g} / \mathrm{cm}^{3}$
No TRWPs
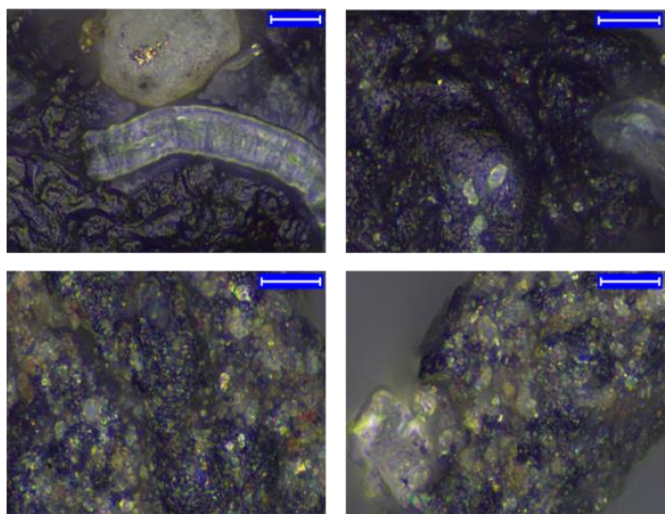

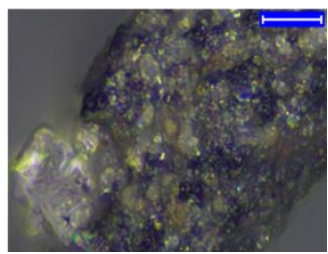

Figure 9. Magnified surface images of the TRWPs classified from the road samples. The blue scale bar is $10 \mu \mathrm{m}$.

There were rare TRWPs in the RD2 sample $(212-500 \mu \mathrm{m})$, but their aspect ratios were very different from each other, as listed in Table 2. Only 3 TRWPs were found in the RD2 sample. The L3 TRWP had very long shape and its aspect ratio was over 30. A total of 21 TRWPs were found in the RD3 sample (106-212 $\mu \mathrm{m})$ and the TRWPs were given a number according to the aspect ratio as well as the density (Table 3). There was no specific trend for the aspect ratios with the density. The lowest aspect ratio was 1.22 , while the highest one was 5.21. There were many TRWPs with very low aspect ratios below $2.00 \mathrm{~g} / \mathrm{cm}^{3}$ in the TRWPs of 1.60-1.70 g/ $\mathrm{cm}^{3} .65$ TRWPs were found in the RD4 sample $(63-106 \mu \mathrm{m})$ and they were given a number according to the aspect ratio as well as the density (Table 4 ). The lowest aspect ratio was 1.21, while the highest one was 4.31. Aspect ratios of the TRWPs in the RD4 sample were relatively lower than those in the RD3 sample. There were many TRWPs with very low aspect ratios below 2.00 for all of the density ranges. Ratios of the TRWPs with very low aspect ratios below 2.00 tended to increase by increasing the density. The ratios were 31, 42, 35, and 50\% for the TRWPs with the densities of 1.30-1.40, 1.40-1.50, $1.50-1.60$, and $1.60-1.70 \mathrm{~g} / \mathrm{cm}^{3}$, respectively.

Table 2. Aspect ratios of TRWPs separated from the RD2 sample $(212-500 \mu \mathrm{m})$.

\begin{tabular}{ccccc}
\hline Density $\left(\mathbf{g} / \mathbf{c m}^{3}\right)$ & TRWP No. & Length $(\mu \mathrm{m})$ & Width $(\boldsymbol{\mu m})$ & Aspect Ratio \\
\hline \multirow{2}{*}{$1.20-1.30$} & L1 & 906.818 & 443.243 & 2.046 \\
& L2 & 819.610 & 328.868 & 2.492 \\
\hline \multirow{2}{*}{$1.40-1.50$} & L3 & 2210.560 & 70.730 & 31.253 \\
\hline
\end{tabular}

Py-GC chromatograms of the TRWPs separated from the RD2, RD3, and RD4 samples showed similar pyrolysis products, as shown in Figure 10. The major pyrolysis products were isoprene and dipentene which are the key pyrolysis products of natural rubber (NR) [13-16]. Since bus tire treads are mainly made of NR [17-20], the TRWPs should come from abrasion of bus tire treads. Styrene was one of main pyrolysis products of the TRWPs and this could originate from abrasion of tire tread made of styrene-butadiene rubber (SBR) or APWPs attatched to the TRWPs. Styrene and 4-vinylcyclohexene (VCH) are the key pyrolysis products of SBR [21-23]. VCH is also the key pyrolysis products of 
butadiene rubber (BR) [24]. Hence, the TRWPs should be composed of NR/SBR biblend or NR/BR/SBR triblend compound. The TRWP of the RD2 sample showed very intensive styrene peak but the $\mathrm{VCH}$ peak was relatively small. This implies that some styrene might come from the other source such as APWP not SBR. For the TRWP of the RD3 sample, the peak intensities of styrene and $\mathrm{VCH}$ were nearly the same, which means that BR content in the TRWPs was not lower than SBR sample. 13 TRWPs of the RD3 sample were analyzed together so their rubber compositions might be different each other.

Table 3. Aspect ratios of TRWPs separated from the RD3 sample (106-212 $\mu \mathrm{m})$.

\begin{tabular}{|c|c|c|c|c|}
\hline Density $\left(\mathrm{g} / \mathrm{cm}^{3}\right)$ & TRWP No. & Length $(\mu \mathrm{m})$ & Width $(\mu \mathrm{m})$ & Aspect Ratio \\
\hline \multirow{5}{*}{$1.20-1.30$} & M1 & 213.525 & 102.610 & 2.081 \\
\hline & M2 & 216.658 & 79.177 & 2.736 \\
\hline & M3 & 288.630 & 101.299 & 2.849 \\
\hline & M4 & 212.629 & 64.337 & 3.305 \\
\hline & M5 & 388.050 & 100.524 & 3.860 \\
\hline \multirow{4}{*}{$1.30-1.40$} & M6 & 81.069 & 49.217 & 1.647 \\
\hline & M7 & 97.367 & 45.208 & 2.154 \\
\hline & M8 & 207.638 & 91.421 & 2.271 \\
\hline & M9 & 354.829 & 68.065 & 5.213 \\
\hline \multirow{2}{*}{$1.40-1.50$} & M10 & 142.078 & 57.064 & 2.490 \\
\hline & M11 & 744.581 & 189.547 & 3.928 \\
\hline \multirow{2}{*}{$1.50-1.60$} & M12 & 176.207 & 97.506 & 1.807 \\
\hline & M13 & 387.485 & 104.674 & 3.702 \\
\hline \multirow{8}{*}{$1.60-1.70$} & M14 & 84.341 & 69.123 & 1.220 \\
\hline & M15 & 119.108 & 68.880 & 1.729 \\
\hline & M16 & 135.348 & 76.499 & 1.769 \\
\hline & M17 & 155.191 & 86.731 & 1.789 \\
\hline & M18 & 173.171 & 86.788 & 1.995 \\
\hline & M19 & 167.619 & 69.844 & 2.400 \\
\hline & M20 & 152.797 & 48.497 & 3.151 \\
\hline & M21 & 166.919 & 43.192 & 3.865 \\
\hline
\end{tabular}

Table 4. Aspect ratios of TRWPs separated from the RD4 sample $(63-106 \mu \mathrm{m})$.

\begin{tabular}{ccccc}
\hline Density $\left(\mathbf{g} / \mathbf{c m}^{\mathbf{3}}\right)$ & TRWP No. & Length $(\boldsymbol{\mu m})$ & Width $(\boldsymbol{\mu m})$ & Aspect Ratio \\
\hline & S1 & 75.987 & 62.774 & 1.210 \\
S2 & 84.482 & 69.276 & 1.219 \\
S3 & 104.769 & 66.094 & 1.585 \\
S4 & 102.466 & 58.350 & 1.756 \\
& S5 & 85.681 & 40.413 & 2.120 \\
$1.30-1.40$ & S6 & 89.423 & 41.858 & 2.136 \\
& S7 & 143.217 & 64.288 & 2.228 \\
& S8 & 106.022 & 42.768 & 2.479 \\
& S9 & 175.500 & 67.418 & 2.603 \\
& S10 & 132.855 & 47.826 & 2.778 \\
& S11 & 122.079 & 42.096 & 2.900 \\
& S12 & 181.297 & 51.182 & 3.542 \\
& S13 & 185.188 & 44.504 & 4.161 \\
\hline
\end{tabular}


Table 4. Cont.

\begin{tabular}{|c|c|c|c|c|}
\hline Density $\left(\mathrm{g} / \mathrm{cm}^{3}\right)$ & TRWP No. & Length $(\mu \mathrm{m})$ & Width $(\mu \mathrm{m})$ & Aspect Ratio \\
\hline \multirow{19}{*}{$1.40-1.50$} & S14 & 68.380 & 51.267 & 1.334 \\
\hline & S15 & 51.300 & 36.674 & 1.399 \\
\hline & S16 & 74.494 & 50.048 & 1.488 \\
\hline & S17 & 70.844 & 46.010 & 1.540 \\
\hline & S18 & 79.489 & 50.976 & 1.559 \\
\hline & S19 & 71.347 & 45.476 & 1.569 \\
\hline & S20 & 70.813 & 44.320 & 1.598 \\
\hline & S21 & 74.040 & 37.798 & 1.959 \\
\hline & $\mathrm{S} 22$ & 96.020 & 46.071 & 2.084 \\
\hline & $\mathrm{S} 23$ & 87.544 & 41.531 & 2.108 \\
\hline & S24 & 75.285 & 34.674 & 2.171 \\
\hline & $\mathrm{S} 25$ & 95.998 & 43.113 & 2.227 \\
\hline & S26 & 88.719 & 38.018 & 2.334 \\
\hline & $\mathrm{S} 27$ & 69.131 & 26.746 & 2.585 \\
\hline & S28 & 92.232 & 30.691 & 3.005 \\
\hline & S29 & 87.772 & 27.016 & 3.249 \\
\hline & S30 & 160.384 & 41.452 & 3.869 \\
\hline & S31 & 244.556 & 62.589 & 3.907 \\
\hline & S32 & 75.939 & 17.631 & 4.307 \\
\hline \multirow{17}{*}{$1.50-1.60$} & S33 & 78.593 & 53.737 & 1.463 \\
\hline & S34 & 104.537 & 70.370 & 1.486 \\
\hline & S35 & 58.257 & 37.340 & 1.560 \\
\hline & S36 & 110.743 & 65.747 & 1.684 \\
\hline & S37 & 71.824 & 39.602 & 1.814 \\
\hline & S38 & 80.917 & 41.093 & 1.969 \\
\hline & S39 & 76.901 & 31.860 & 2.414 \\
\hline & $\mathrm{S} 40$ & 93.174 & 36.110 & 2.580 \\
\hline & S41 & 159.109 & 54.829 & 2.902 \\
\hline & S42 & 126.799 & 42.858 & 2.959 \\
\hline & $\mathrm{S} 43$ & 150.389 & 48.767 & 3.084 \\
\hline & S44 & 112.468 & 34.436 & 3.266 \\
\hline & S45 & 139.460 & 40.920 & 3.408 \\
\hline & S46 & 111.306 & 32.553 & 3.419 \\
\hline & S47 & 105.397 & 30.033 & 3.509 \\
\hline & S48 & 121.955 & 34.548 & 3.530 \\
\hline & S49 & 168.570 & 47.300 & 3.564 \\
\hline \multirow{16}{*}{$1.60-1.70$} & S50 & 150.119 & 106.791 & 1.406 \\
\hline & S51 & 100.285 & 68.619 & 1.461 \\
\hline & S52 & 109.567 & 72.483 & 1.512 \\
\hline & S53 & 210.149 & 131.425 & 1.599 \\
\hline & S54 & 183.867 & 107.128 & 1.716 \\
\hline & S55 & 99.901 & 53.580 & 1.865 \\
\hline & S56 & 82.631 & 42.693 & 1.935 \\
\hline & S57 & 97.152 & 48.720 & 1.994 \\
\hline & S58 & 152.058 & 74.227 & 2.049 \\
\hline & S59 & 107.015 & 50.644 & 2.113 \\
\hline & S60 & 194.690 & 84.565 & 2.302 \\
\hline & S61 & 83.901 & 35.866 & 2.339 \\
\hline & S62 & 127.199 & 49.838 & 2.552 \\
\hline & S63 & 123.673 & 48.075 & 2.573 \\
\hline & S64 & 285.707 & 75.658 & 3.776 \\
\hline & S65 & 364.601 & 91.268 & 3.995 \\
\hline
\end{tabular}




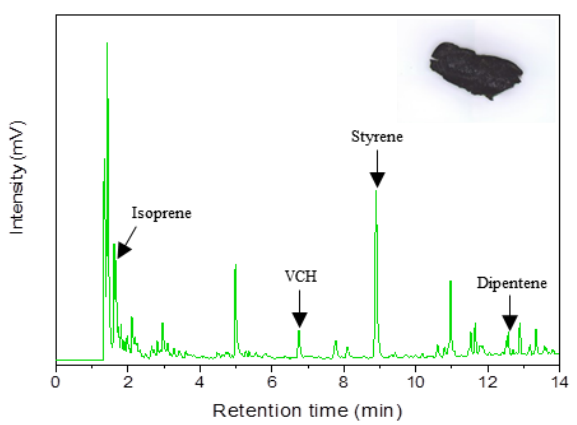

(a)
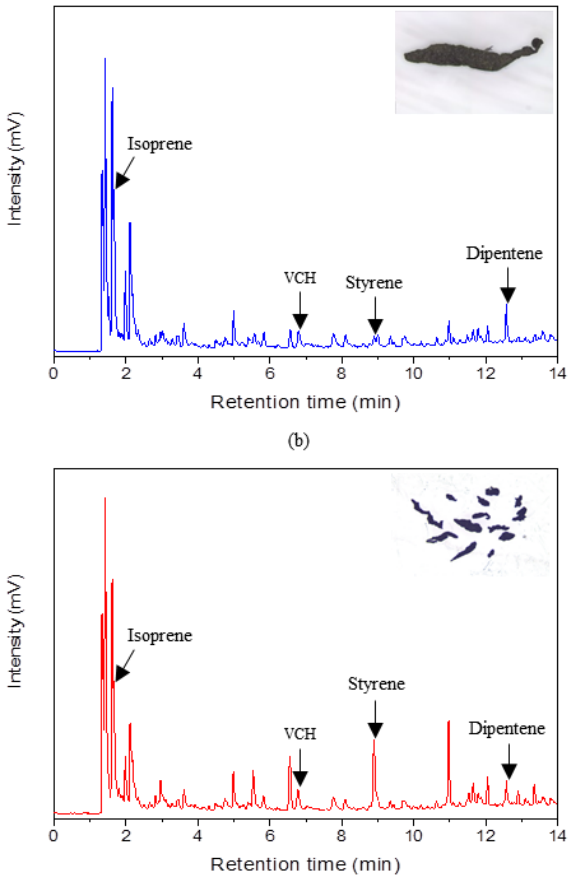

Figure 10. Py-GC chromatograms of the TRWPs classified from the road dust samples. (a) the RD2 sample (single TRWP, $1.20-1.30 \mathrm{~g} / \mathrm{cm}^{3}$ ), (b) the RD3 sample (single TRWP, $1.40-1.50 \mathrm{~g} / \mathrm{cm}^{3}$ ), and (c) the RD4 sample (13 TRWPs, $\left.1.30-1.40 \mathrm{~g} / \mathrm{cm}^{3}\right)$.

\section{Conclusions}

Based on the density ranges of TRWPs, the density separation was carried out using the salt solutions with different densities of $1.20,1.30,1.40,1.50,1.60$, and $1.70 \mathrm{~g} / \mathrm{cm}^{3}$. TRWPs were differentiated from APWPs in the same density range using chloroform treatment. TRWPs were classified into 5 groups according to the density ranges of $1.20-1.30$, $1.30-1.40,1.40-1.50,1.50-1.60$, and $1.60-1.70 \mathrm{~g} / \mathrm{cm}^{3}$. There was no TRWP below $1.20 \mathrm{~g} / \mathrm{cm}^{3}$ or above $1.70 \mathrm{~g} / \mathrm{cm}^{3}$. For the RD2 sample $(212-500 \mu \mathrm{m})$, TRWPs were rarely found. For the RD3 sample (106-212 $\mu \mathrm{m})$, TRWPs with all density ranges of $1.20-1.70 \mathrm{~g} / \mathrm{cm}^{3}$ were found. There were relatively even TRWPs with density ranges of $1.30-1.70 \mathrm{~g} / \mathrm{cm}^{3}$ in the RD4 sample (63-106 $\mu \mathrm{m})$. For the TRWPs with the same density range, the TRWP content tended to increase with decrease in the particle size. Sizes of the tiny particles adsorbed on the TRWP surface varied and the number of the tiny particles tended to increase as the TRWP density increased. There was no specific trend for the aspect ratios with the TRWP density. Almost TRWPs were in the aspect ratio range of 1.2-5.2. Percentage of the TRWPs with very low aspect ratios below 2.00 tended to increase by increasing the TRWP density. Major pyrolysis products of the TRWPs were isoprene and dipentene which means that the TRWPs were mainly made of NR and they came from the abrasion of bus tire treads. 
Styrene and VCH were also observed as the main pyrolysis products, meaning that the TRWP might be composed of NR/SBR biblend or NR/BR/SBR triblend compound.

Author Contributions: U.J.: road dust collection \& separation, density separation, formal analysis, data curation, visualization, writing — first draft. S.-S.C.: resources, supervision, writing-original draft \& editing. All authors have read and agreed to the published version of the manuscript.

Funding: This work was supported by the Technology Innovation Program funded by the Ministry of Trade, Industry and Energy, Korea (Project Number 20010851).

Institutional Review Board Statement: Not applicable.

Informed Consent Statement: Not applicable.

Data Availability Statement: The data presented in this study are available on request from the corresponding author.

Conflicts of Interest: The authors declare no conflict of interest.

\section{References}

1. Kovochich, M.; Parker, J.A.; Oh, S.C.; Lee, J.P.; Wagner, S.; Reemtsma, T.; Unice, K.M. Characterization of individual tire and road wear particles in environmental road dust, tunnel dust, and sediment. Environ. Sci. Technol. Lett. 2021, 8, 1057-1064. [CrossRef]

2. Klockner, P.; Reemtsma, T.; Eisentraut, P.; Braun, U.; Ruhl, A.S.; Wagner, S. Tire and road wear particles in road environmentQuantification and assessment of particle dynamics by Zn determination after density separation. Chemosphere 2019, 222, 714-721. [CrossRef] [PubMed]

3. $\quad$ Kreider, M.L.; Panko, J.M.; McAtee, B.L.; Sweet, L.I.; Finley, B.L. Physical and chemical characterization of tire-related particles: Comparison of particles generated using different methodologies. Sci. Total Environ. 2010, 408, 652-659. [CrossRef]

4. Jung, U.Y.; Choi, S.S. A variety of particles including tire wear particles produced on the road. Elast. Compos. 2021, 56, 85-91.

5. Sakai, H. Friction and wear of tire tread rubber. Tire Sci. Technol. 1996, 24, 252-275. [CrossRef]

6. Kole, P.J.; Löhr, A.J.; Van Belleghem, F.G.A.J.; Ragas, A.M.J. Wear and tear of tyres: A stealthy source of microplastics in the environment. Int. J. Environ. Res. Public Health 2017, 14, 1265. [CrossRef]

7. Järlskog, I.; Strömvall, A.-M.; Magnusson, K.; Gustafsson, M.; Polukarova, M.; Galfi, H.; Aronsson, M.; Andersson-Sköld, Y. Occurrence of tire and bitumen wear microplastics on urban streets and in sweepsand and washwater. Sci. Total Environ. 2020, 729, 138950. [CrossRef]

8. Järlskog, I.; Strömvall, A.-M.; Magnusson, K.; Galfi, H.; Björklund, K.; Polukarova, M.; Garção, R.; Markiewicz, A.; Aronsson, M.; Gustafsson, M.; et al. Traffic-related microplastic particles, metals, and organic pollutants in an urban area under reconstruction. Sci. Total Environ. 2021, 774, 145503. [CrossRef]

9. Degaffe, F.S.; Turner, A. Leaching of zinc from tire wear particles under simulated estuarine conditions. Chemosphere 2011, 85, 738-743. [CrossRef]

10. Unice, K.M.; Weeber, M.P.; Abramson, M.M.; Reid, R.C.D.; Gils, J.A.G.V.; Markus, A.A.; Vethaak, A.D.; Panko, J.M. Characterizing export of land-based microplastics to the estuary-Part I: Application of integrated geospatial microplastic transport models to assess tire and road wear particles in the Seine watershed. Sci. Total Environ. 2019,646, 1639-1649. [CrossRef]

11. Lan, H.; Martin, C.D.; Hu, B. Effect of heterogeneity of brittle rock on micromechanical extensile behavior during compression loading. J. Geophys. Res. Solid Earth 2010, 115, B01202. [CrossRef]

12. Sarpün, İ.H.; Kılıçkaya, M.S.; Tuncel, S. Mean grain size determination in marbles by ultrasonic velocity techniques. NDT Int. 2005, 38, 21-25. [CrossRef]

13. Januszewicz, K.; Kazimierski, P.; Suchocki, T.; Kardas, D.; Lewandowski, W.; Klugmann-Radziemska, E.; Luczak, J. Waste rubber pyrolysis: Product yields and limonene concentration. Materials 2020, 13, 4435. [CrossRef] [PubMed]

14. Danon, B.; van der Gryp, P.; Schwarz, C.E.; Görgens, J.F. A review of dipentene (DL-limonene) production from waste tire pyrolysis. J. Anal. Appl. Pyrolysis 2015, 112, 1-13. [CrossRef]

15. Choi, S.-S.; Ko, J.-E. Influence of thermal aging on pyrolysis pattern of carbon black-filled NR composite. Macromol. Res. 2007, 15, 482-485. [CrossRef]

16. Pakdel, H.; Pantea, D.M.; Roy, C. Production of $d l$-limonene by vacuum pyrolysis of used tires. J. Anal. Appl. Pyrolysis 2001, 57, 91-107. [CrossRef]

17. Chae, E.; Jung, U.; Choi, S.-S. Quantification of tire tread wear particles in microparticles produced on the road using oleamide as a novel marker. Environ. Pollut. 2021, 288, 117811. [CrossRef] [PubMed]

18. Choi, S.-S.; Yang, S.R.; Chae, E.; Son, C.E. Influence of carbon black contents and rubber compositions on formation of wear debris of rubber vulcanizates. Elast. Compos. 2020, 55, 108-113.

19. Miyazaki, T. Rubber Composition. European Patent EP3459996, 21 April 2017.

20. Ghoreishy, M.H.R.; Alimardani, M.; Mehrabian, R.Z.; Gangali, S.T. Modeling the hyperviscoelastic behavior of a tire tread compound reinforced by silica and carbon black. J. Appl. Polym. Sci. 2013, 128, 1725-1731. 
21. Unice, K.M.; Kreider, M.L.; Panko, J.M. Use of a deuterated internal standard with pyrolysis-GC/MS dimeric marker analysis to quantify tire tread particles in the environment. Int. J. Environ. Res. Public Health 2012, 9, 4033-4055. [CrossRef]

22. Seidelt, S.; Müller-Hagedorn, M.; Bockhorn, H. Description of tire pyrolysis by thermal degradation behaviour of main components. J. Anal. Appl. Pyrolysis 2006, 75, 11-18. [CrossRef]

23. Choi, S.-S. Characteristics of pyrolysis pattern of styrene-butadiene rubbers with differing microstructures. J. Anal. Appl. Pyrolysis 2002, 62, 319-330. [CrossRef]

24. Choi, S.-S.; Han, D.-H. Pyrolysis paths of polybutadiene depending on pyrolysis temperature. Macromol. Res. 2006, 14, 354-358. [CrossRef] 